\title{
Comparison of Confinement and Loss of Plasmonic Waveguides
}

\author{
X. Sun, M. Z. Alam, J. S. Aitchison and M. Mojahedi \\ Department of Electrical \& Computer Engineering, University of Toronto \\ Toronto, Ontario, Canada M5S3G4 \\ xiaoxiao.sun@mail.utoronto.ca
}

\begin{abstract}
We have compared a number of well known plasmonic guides in terms of power confinement, normalized power density, and propagation loss. We have identified the relative advantages and limitations of these guides.
\end{abstract}

Keywords-surface plasmon; integrated optics; propagation loss; mode confinement.

\section{INTRODUCTION}

In recent years plasmonic wave guiding has emerged as a very attractive guiding mechanism because of its ability to confine light in a very small area. Many different kinds of plasmonic waveguides have been proposed [1-4] and many applications have been suggested $[5,6]$. For realization of the full potential of plasmonics, it is important to compare different plasmonic waveguides and identify the best waveguide design for various applications. A combination of low propagation loss, high power density, and large confinement will be useful for many applications of plasmonic waveguides including nonlinear optics [5] and biosensing [6]. In this work we compare some popular plasmonic waveguides in these regards and try to identify their relative advantages and limitations.

\section{ANALYZED WAVEGUide StRUCtURES AND Method OF COMPARISION}

Some of the most popular plasmonic guides proposed for nanophotonic applications are dielectric loaded surface plasmon waveguide (DLSPW) [1], plasmonic slot waveguide [2], and hybrid plasmonic waveguide (HPWG) $[3,4]$. The geometries of these guides are shown in Fig. 1 (ac). We intend to compare the relative performance of these guides and also compare their performance with the silicon nanowire waveguide shown in Fig. 1(d).

Unlike dielectric waveguides for which the propagation loss can be negligible, the presence of metal as part of the guiding structure makes plasmonic guides highly lossy. In general, any plasmonic guide exhibits a tradeoff between propagation loss and mode confinement - the smaller the mode size, the higher the propagation loss. Unlike a typical dielectric waveguide whose guided mode has a circular or elliptical shape, the shapes of the modes supported by plasmonic guides are usually more complicated. As a result evaluation of mode confinement of plasmonic guides is a challenging task. This issue has been discussed in $[7,8]$ and it asserted that the definition of mode area should depends on the specific application. The common way of expressing a waveguide's ability to confine light is to calculate its mode size, and various definitions of mode size have been used by the plasmonics research community. Although the mode size can be a good indication of a guide's ability to confine light, it is not sufficient in many cases. Many applications of plamsonics like nonlinear optics [5] and biosensing [6] aim at utilizing the high power density of plasmonic guides. For such applications, in addition to having a small mode size, it is also important to confine the power in a specific area of the wave guiding region. For example, a typical application of DLSPW would probably require power confinement in the high index core marked by the dotted line in Fig. 1(a). Application of slot waveguide on the other hand will most likely require the power to be concentrated in the slot region indicated by the dotted line in Fig. 1(b). Typical applications of HPWG would require high power concentration in the region between the silicon and metal indicated by the dotted line in Fig. 1(c). It is unlikely one would intend to use HPWG for confining light in the high index medium which can be achieved in much simpler manner as shown in Figs. 1 (a) and 1 (d).
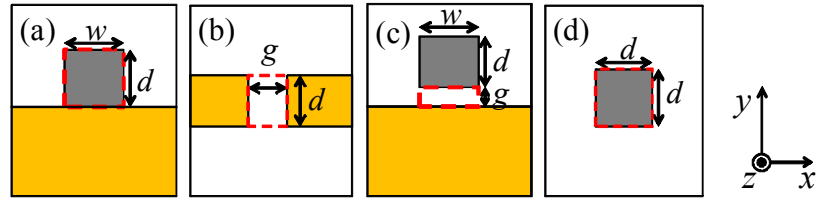

Fig.1 (a) Dielectric loaded surface plasmon waveguide, (b) Plasmonic slot waveguide, (c) Hybrid plasmonic waveguide, (d) Silicon waveguide.

In this work, to compare different types of plasmonic guides, instead of defining mode size as in [7] or figure of merit as in [8], we use two other criterions: power confinement $(\Gamma)$ in the area of interest and power density $(D)$ in the area of interest. For the power propagating in the waveguide along the $z$-direction, confinement factor $\Gamma$ is defined as the fraction of power confined in region $i$ [area of interest marked by the dotted lines in Figs. 1(a) to 1(d)] according to

$$
\Gamma=\frac{\iint_{i}\left|<S_{z}(x, y)>\right| d x d y}{\int_{-\infty}^{\infty} \int_{-\infty}^{\infty}\left|<S_{z}(x, y)\right\rangle \mid d x d y} .
$$

Here, $S_{z}$ is the time-averaged pointing vector along the $z$ direction.

The confinement factor alone is not enough to evaluate the usefulness of a waveguide. In addition to having a large $\Gamma$ it may also important to have high power density $D$ in the area of interest. To evaluate this we define the power density $D$ in the following manner

$$
D=\frac{\Gamma \cdot P_{\text {total }}}{A_{i}}
$$


Here, $A_{i}$ is the area enclosed by the dotted lines in Figs. 1(a)(d) and $P_{\text {total }}$ is the total guided power, which is normalized to 1 in the present case. In the following we investigate which of the plasmonic guides can provide large values of $\Gamma$ and $D$ for the same propagation loss.

\section{RESULTS}

We use commercial finite difference software Lumerical Mode Solutions to analyze the effects of waveguide dimensions on the performance of the guide. The wavelength of operation is $1.55 \mu \mathrm{m}$ and properties of silver are taken from [9]. Silicon waveguide shown in Fig.1 (d) is chosen as the reference. For the silicon guide the highest $D$ is about 7.8 $/ \mu \mathrm{m}^{2}$ which is achieved for $d=300 \mathrm{~nm}$ and the corresponding $\Gamma$ is $70 \%$.

Figure 2 shows the power density achievable for the plasmonic waveguides shown in Fig. 1. We have optimized each of the waveguides to achieve high power density, good confinement, and low propagation loss. For conciseness we report only the best cases for each waveguide. The arrows in Fig. 2 indicate increasing dimensions ( $d$ or $g$ ). DLSPW consists of a finite width dielectric film deposited on a metal film. We chose to examine DLSPW consisting of a silicon slab on a metal surface since it can provide very high confinement [1]. For the DLSPW the width $(w)$ is fixed at $200 \mathrm{~nm}$ and the height of the silicon layer $(d)$ is varied from $150 \mathrm{~nm}$ to $300 \mathrm{~nm}$. The corresponding $D$ for the DLSPW varies from $13 / \mu \mathrm{m}^{2}$ to $24 / \mu \mathrm{m}^{2}$. For the slot waveguide, the metal thickness $(d)$ is fixed at $100 \mathrm{~nm}$ and the slot width $(g)$ is varied from 30 to $100 \mathrm{~nm}$. This guide can provide the highest power density which can be approximately $200 / \mu^{2}$. For the HPWG, both the width $(w)$ and silicon thickness $(d)$ have been fixed at $200 \mathrm{~nm}$ and the gap $(g)$ is varied from 10 to $100 \mathrm{~nm}$. The power density achievable for the HPWG is higher than the DLSPW but lower compared to the slot waveguide. However, for a given power density the propagation loss of the HPWG is much lower compared to the other types of plasmonic guides.

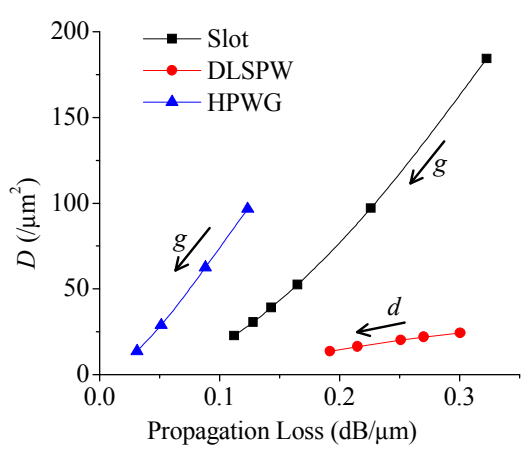

Fig. 2 Normalized power density vs. propagation loss for three types of plasmonic waveguides.

Figure 3 shows the confinement factor achievable for the plasmonic guides under consideration. The dimensions chosen are same as those in Fig. 2. The confinement is best for the DLSPW (85\%) and comparable to that achievable from silicon nanowire waveguide. The confinement is relatively low for the HPWG compared to the other guides but its propagation loss is also much lower.

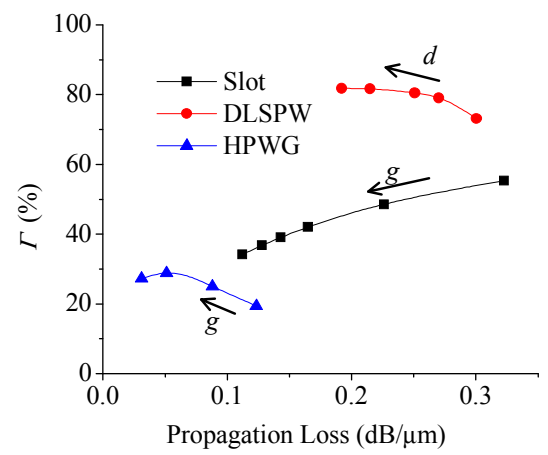

Fig. 3 Confinement factor vs. propagation loss for three types of plasmonic waveguides.

From the analysis above we observe that the performance of the DLSPW is very similar to the silicon waveguide, but it suffers from a very high propagation loss. Therefore, DLSPW may not be a suitable choice for applications where high power density is required, nonlinear optics for example. The slot waveguide can achieve very high power density and good confinement but the propagation loss is also very high. HPWG is a compromise in the sense that it has significantly lower loss but still can provide high power density and moderate confinement. The choice between HPWG and slot waveguides should depend on the relative importance of power density and power confinement for a specific application.

\section{REFERENCES}

[1] A. V. Krasavin and A.V. Zayats, "Silicon-based plasmonic waveguides," Opt. Express, Vol. 18, pp. 11791-11799, 2010.

[2] G. Veronis and S. Fan, "Guided subwavelength plasmonic mode supported by a slot in a thin metal film," Opt. Lett., Vol. 30, pp. 3359-3361, 2005.

[3] M. Z. Alam, J. Meier, J. S. Aitchison, and M. Mojahedi, "Super mode propagation in low index medium," CLEO conference, Paper ID JThD112, 2007.

[4] M. Z. Alam, J. Meier, J. S. Aitchison, M. Mojahedi, "Propagation characteristics of hybrid modes supported by metal-low-high index waveguides and bends", Opt. Express, Vol. 18, pp. 12971-12979, 2010 .

[5] F. Lu, T. Li, J. Xu, Z. Xie, L. Li, S. Zhu, and Y. Zhu, "Surface plasmon polariton enhanced by optical parametric amplification in nonlinear hybrid waveguide," Opt. Express, Vol. 19, pp. 2858-2865, 2011.

[6] A. Shalabney and I. Abdulhalim, "Electromagnetic fields distribution in multilayer thin film structures and the origin of sensitivity enhancement in surface plasmon resonance sensors," Sensors Actuators A: Physical, Vol. 159, pp. 24-32, 2010.

[7] R. F. Oulton, G. Bartal, D. F. P. Pile, and X. Zhang, "Confinement and propagation characterisics of subwavelength plasmonic modes," New J. of Phys. Vol. 10, 105018, 2008.

[8] R. Buckley and P. Berini, "Figures of merit for 2D surface plasmon waveguides and application to metal stripes," Opt. Express, Vol. 15, pp. 12174-12182, 2007.

[9] P. B. Johnson and R. W. Christy, "Optical constants of the noble metals,” Phys. Rev. B, Vol. 6, pp. 4370-4379, 1972. 